\title{
Binet - Type Formula For The Sequence of Tetranacci Numbers by Alternate Methods
}

\section{GAUTAM S. HATHIWALA AND DEVBHADRA V. SHAH}

C.K. Pithawala College of Enigneering \& Technology, Surat.

Department of Mathematics, Veer Narmad South Gujarat University, Surat.

\section{Email: gautamhathiwala1717@gmail.com}

Received: June 23, 2017 | Revides: July 02, 2017 | Accepted: August 06, 2017

Published Online: September 01, 2017

The Author(s) 2017. This article is published with open access at www.chitkara.edu.in/publications

Abstract The sequence $\left\{T_{n}\right\}$ of Tetranacci numbers is defined by the recurrence relation $T_{n}=T_{n-1}+T_{n-2}+T_{n-3}+T_{n-4} ; n \geq 4$ with initial condition $T_{0}=T_{1}=T_{2}=0$ and $T_{3}=1$. In this paper, we obtain the explicit formula Binet - type formula for $T_{n}$ by two different methods. We use the concept of eigen decomposition as well as of generating functions to obtain the result.

Keywords: Binet formula, Fibonacci sequence, Tetranacci sequence.

Mathematics Subject Classification: 11B39, 15B36.

\section{INTRODUCTION}

Fibonacci sequence is a sequence of numbers defined by the recursive formula $F_{n}=F_{n-1}+F_{n-2}$; for $n>2$ with initial condition $F_{1}=1, F_{2}=1$. This sequence possesses many interesting properties which have been studied in detail ([8], [12]). Analogous to Fibonacci sequence, many other sequences have been defined, either by changing the initial terms or the recursive relation or both, to obtain the new sequence which may possess similar properties ([9], [11]). One of the important result that is associated with Fibonacci numbers and which has been studied for centuries now, is the Binet formula given as $F_{n}=\frac{\phi^{n}-(-\phi)^{-n}}{\sqrt{5}}$; where $\phi=\frac{1+\sqrt{5}}{2}$ and $F_{n}$ is the $n^{\text {th }}$ Fibonacci number.

Also, by changing the recursive formula or the initial terms, we can have a new sequence of numbers defined and correspondingly, a new Binet - type formula

Mathematical Journal of Interdisciplinary Sciences Vol-6, No-1, September 2017 pp. $37-48$ 
Hathiwala, GS Shah, DV

can be obtained ([2], [6], [7]). In this paper we consider the sequence of Tetranacci numbers and obtain the Binet - type formula for by two different methods.

Definition: The sequence of Tetranacci numbers is defined by the recurrence relation $T_{n}=T_{n-1}+T_{n-2}+T_{n-3}+T_{n-4}$; for $n \geq 4$ with initial terms $T_{0}=T_{1}=T_{2}=0$ and $T_{3}=1$. The first few terms of the sequence $\left\{T_{n}\right\}$ are.

$$
0,0,0,1,1,2,4,8,15,29,56,108,208,401,773,1490,2872 \ldots .
$$

Many salient features of this sequence have been studied in detail ([4], [5], [10]).

\section{PRELIMINARY RESULTS}

In this section, we give some preliminary results which will be useful to derive the Binet - type formula for .

Proposition 2.1 $\left[\begin{array}{c}T_{n} \\ T_{n+1} \\ T_{n+2} \\ T_{n+3}\end{array}\right]=\left[\begin{array}{llll}0 & 1 & 0 & 0 \\ 0 & 0 & 1 & 0 \\ 0 & 0 & 0 & 1 \\ 1 & 1 & 1 & 1\end{array}\right]^{n}\left[\begin{array}{l}0 \\ 0 \\ 0 \\ 1\end{array}\right]$.

Proof: Above result can be proved using principal of mathematical induction. It is clearly observed that result is true for $n=1$. Let it be true for some positive integer $k$. This gives,

$$
\left[\begin{array}{c}
T_{k} \\
T_{k+1} \\
T_{k+2} \\
T_{k+3}
\end{array}\right]=\left[\begin{array}{llll}
0 & 1 & 0 & 0 \\
0 & 0 & 1 & 0 \\
0 & 0 & 0 & 1 \\
1 & 1 & 1 & 1
\end{array}\right]^{k}\left[\begin{array}{l}
0 \\
0 \\
0 \\
1
\end{array}\right]
$$

Now consider $n=k+1$. Then we have

$$
\text { R.H.S. }=\left[\begin{array}{llll}
0 & 1 & 0 & 0 \\
0 & 0 & 1 & 0 \\
0 & 0 & 0 & 1 \\
1 & 1 & 1 & 1
\end{array}\right]^{k+1}\left[\begin{array}{l}
0 \\
0 \\
0 \\
1
\end{array}\right]=\left[\begin{array}{llll}
0 & 1 & 0 & 0 \\
0 & 0 & 1 & 0 \\
0 & 0 & 0 & 1 \\
1 & 1 & 1 & 1
\end{array}\right]\left[\begin{array}{llll}
0 & 1 & 0 & 0 \\
0 & 0 & 1 & 0 \\
0 & 0 & 0 & 1 \\
1 & 1 & 1 & 1
\end{array}\right]^{k}\left[\begin{array}{l}
0 \\
0 \\
0 \\
1
\end{array}\right] .
$$


Using the induction hypothesis, we get

$$
\text { R.H.S. }=\left[\begin{array}{llll}
0 & 1 & 0 & 0 \\
0 & 0 & 1 & 0 \\
0 & 0 & 0 & 1 \\
1 & 1 & 1 & 1
\end{array}\right]^{k+1}\left[\begin{array}{l}
0 \\
0 \\
0 \\
1
\end{array}\right]=\left[\begin{array}{llll}
0 & 1 & 0 & 0 \\
0 & 0 & 1 & 0 \\
0 & 0 & 0 & 1 \\
1 & 1 & 1 & 1
\end{array}\right]\left[\begin{array}{c}
T_{k} \\
T_{k+1} \\
T_{k+2} \\
T_{k+3}
\end{array}\right]=\left[\begin{array}{c}
T_{k+1} \\
T_{k+2} \\
T_{k+3} \\
T_{k+4}
\end{array}\right]=\text { L.H.S. }
$$

Thus result is true for $n=k+1$ also, and hence for all $n$.

We next derive the generating function for the sequence $\left\{T_{n}\right\}$.

Proposition 2.2: $t(x)=\frac{x^{3}}{1-x-x^{2}-x^{3}-x^{4}}$.

Proof: Let $t(x)$ bethepolynomialofinfinitedegree withitscoefficients as Tetranacci numbers. i.e. let $t(x)=\sum_{n=0}^{\infty} T_{n} x^{n}=T_{0}+T_{1} x+T_{2} x^{2}+T_{3} x^{3}+T_{4} x^{4}+T_{5} x^{5}+\ldots$ Now, multiplying this polynomial by $(-x),\left(-x^{2}\right),\left(-x^{3}\right)$ and $\left(-x^{4}\right)$ successively and adding them, we get

$$
\begin{aligned}
\left(1-x-x^{2}-x^{3}-x^{4}\right) t(x)= & T_{0}+\left(T_{1}-T_{0}\right) x+\left(T_{2}-T_{1}-T_{0}\right) x^{2} \\
& +\left(T_{3}-T_{2}-T_{1}-T_{0}\right) x^{3}+\left(T_{4}-T_{3}-T_{2}-T_{1}-T_{0}\right) x^{4}+\ldots
\end{aligned}
$$

This gives, $\left(1-x-x^{2}-x^{3}-x^{4}\right) t(x)=x^{3} \Rightarrow t(x)=\frac{x^{3}}{1-x-x^{2}-x^{3}-x^{4}}$, as
required.

We use this result to derive the Binet - type formula for $T_{n}$. We first use the concept of eigen decomposition of a matrix to derive this formula and then we use the theory of generating functions to obtain the explicit formula for $T_{n}$.

\section{MAIN RESULT}

\section{Theorem 3.1:}

$$
\begin{aligned}
T_{n} & =\frac{\alpha^{n}}{(\alpha-\beta)(\alpha-\gamma)(\alpha-\delta)}+\frac{\beta^{n}}{(\beta-\alpha)(\beta-\gamma)(\beta-\delta)} \\
& +\frac{\gamma^{n}}{(\gamma-\alpha)(\gamma-\beta)(\gamma-\delta)}+\frac{\delta^{n}}{(\delta-\alpha)(\delta-\beta)(\delta-\gamma)}
\end{aligned}
$$

where $\alpha=1.927562, \beta=-0.774804, \gamma=-0.076379+0.8147 i$ and $\delta=\bar{\gamma}$. Proof: By proposition 2.2, we have $t(x)=\frac{x^{3}}{1-x-x^{2}-x^{3}-x^{4}}$. Let $f(x)=1-x-x^{2}-x^{3}-x^{4}$. Then for some $\alpha, \beta, \gamma$ and $\delta$, we write $f(x)=(1-\alpha x)(1-\beta x)(1-\gamma x)(1-\delta x)$. 
Hathiwala, GS Shah, DV

Thus, $\frac{1}{\alpha}, \frac{1}{\beta}, \frac{1}{\gamma}$ and $\frac{1}{\delta}$ are the roots of $f(x)$. This gives $\alpha, \beta, \gamma$ and $\delta$ as the roots of

$$
f\left(\frac{1}{x}\right)=1-\frac{1}{x}-\frac{1}{x^{2}}-\frac{1}{x^{3}}-\frac{1}{x^{4}}=0 .
$$

This implies $x^{4}-x^{3}-x^{2}-x-1=0$. We now solve this equation using Ferrari's method. Consider the substitution $x=\left(y+\frac{1}{4}\right)$. This converts the above equation into depressed quartic form $y^{4}-\frac{11}{2^{3}} y^{2}-\frac{13}{2^{3}} y-\frac{339}{2^{8}}=0$,
which can be written as

$$
\left(y^{2}-\frac{11}{2^{4}}\right)^{2}=\frac{13}{2^{3}} y+\frac{5 \times 23}{2^{6}} .
$$

Next we introduce a new variable $m$ on the L.H.S. of (1) by adding $2 y^{2} m-\frac{11}{2^{3}} m+m^{2}$ on both sides. Now regrouping the powers of $y$ on the R.H.S. of (1), above equation can be transformed as

$$
\left(y^{2}+\frac{11}{2^{4}}+m\right)^{2}=2 m y^{2}+\frac{13}{2^{3}} y+m^{2}-\frac{11}{2^{3}} m+\frac{5 \times 23}{2^{6}} .
$$

We note that (1) and (2) are equivalent for any value of $m$. We select such a value of $m$ which makes R.H.S. of (2) a perfect square. Thus the discriminant in $y$ of this quadratic equation is zero or in other words $m$ is the root of the equation $\left(\frac{13}{2^{3}}\right)^{2}-4(2 m)\left(m^{2}-\frac{11}{2^{3}} m+\frac{5 \times 23}{2^{6}}\right)=0$. On simplification, we get

$$
8 m^{3}-11 m^{2}+\frac{5 \times 23}{2^{3}} m-\frac{13^{2}}{2^{6}}=0
$$

Equation (3) is the resolvent cubic equation of the original quartic equation. We next apply Cardan's method to solve equation (3). For that we take the substitution $m=t+\frac{11}{24}$ and on simplification get the depressed cubic equation as $t^{3}+\frac{7}{6} t+\frac{65}{2^{3} \times 3^{3}}=0$. 
Now we introduce two new variables $i$ and $j$, such that $i+j=t$. Using this in above equation, we have $i^{3}+j^{3}+\left(3 i j+\frac{7}{6}\right)(i+j)+\frac{65}{2^{3} \times 3^{3}}=0$.

Next we impose the condition $3 i j+\frac{7}{6}=0$, which implies $i j=-\frac{7}{18}$.
. This gives

$$
i^{3}+j^{3}=-\frac{65}{2^{3} \times 3^{3}} \text { and } i^{3} j^{3}=-\frac{7^{3}}{2^{3} \times 3^{6}} .
$$

Thus, we get a quadratic equation $z^{2}+\frac{5 \times 13}{2^{3} \times 3^{3}} z-\frac{7^{3}}{2^{3} \times 3^{6}}=0$, whose roots
are $i^{3}$ and $j^{3}$.

We solve this quadratic equation to get the values of $i^{3}$ and $j^{3}$, and eventually values of $i$ and $j$ as

$$
i=-\frac{1}{6} \sqrt[3]{\frac{65-3 \sqrt{1689}}{2}} \text { and } j=-\frac{1}{6} \sqrt[3]{\frac{65+3 \sqrt{1689}}{2}} .
$$

Thus, $t=i+j$ gives $t=-\frac{1}{6 \sqrt[3]{2}}(\sqrt[3]{65-3 \sqrt{1689}}+\sqrt[3]{65+3 \sqrt{1689}})$. This gives,

$$
m=\frac{1}{2^{3} \times 3}\left(11-4\left(\sqrt[3]{\frac{65-3 \sqrt{1689}}{2}}+\sqrt[3]{\frac{65+3 \sqrt{1689}}{2}}\right)\right) .
$$

For this value of $m$, the R.H.S. of equation (2) is of the form $\left(\sqrt{2 m} y+\frac{13}{2^{4} \sqrt{2 m}}\right)^{2}$, which is a perfect square. Thus, from (2), we have $\left(y^{2}+\frac{11}{2^{4}}+m\right)^{2}=\left(\sqrt{2 m} y+\frac{13}{2^{4} \sqrt{2 m}}\right)^{2}$. On solving, we get the four values of $y$ as $1.677561976,-1.024804114,-0.326378931+0.814703648 i$ and $-0.326378931-0.814703648 i$. Since $x=y+\frac{1}{4}$, we get the values of $x$, i.e. the roots of equation $x^{4}-x^{3}-x^{2}-x-1=0$ as $\alpha=1.92756, \beta-0.774804, \gamma=-0.076379+0.8147 i$ and $\delta=-0.076379$ $-0.8147 i$.

Now, using proposition 2.1, we write
Binet - Type Formula For The Sequence of Tetranacci Numbers By Alternate Methods 
Hathiwala, GS

Shah, DV

$$
T_{n}=\left[\begin{array}{llll}
1 & 0 & 0 & 0
\end{array}\right]\left[\begin{array}{llll}
0 & 1 & 0 & 0 \\
0 & 0 & 1 & 0 \\
0 & 0 & 0 & 1 \\
1 & 1 & 1 & 1
\end{array}\right]^{n}\left[\begin{array}{l}
0 \\
0 \\
0 \\
1
\end{array}\right]
$$

Now define $P=\left[\begin{array}{llll}0 & 1 & 0 & 0 \\ 0 & 0 & 1 & 0 \\ 0 & 0 & 0 & 1 \\ 1 & 1 & 1 & 1\end{array}\right]$. We first find the eigenvalues of $P$. For that we consider the characteristic equation as $\operatorname{det}(P-\lambda I)=0$. This gives $\lambda^{4}-\lambda^{3}-\lambda^{2}-\lambda-1=0$. By above discussion it is clear that the roots of this equation are $\alpha, \beta, \gamma$ and $\delta$ and they are the eigenvalues of $P$. Since $P$ has four distinct eigenvalues, it is a diagonalizable matrix [1]. Moreover, for the eigen decomposition of $P$ we find the eigenvectors for the corresponding eigenvalues.

Consider the matrix equation $P x=a x$, where $x$ is the eigenvector corresponding to eigenvalue $\alpha$. This gives $(P-\alpha I) x=\theta$. Solving this matrix equation is equivalent to finding the null - space of $(P-\alpha I)$. Thus, we have

$\left[\begin{array}{cccc}-\alpha & 1 & 0 & 0 \\ 0 & -\alpha & 1 & 0 \\ 0 & 0 & -\alpha & 0 \\ 1 & 1 & 1 & 1-\alpha\end{array}\right]=\theta$. Solving this, we get one of the eigenvector as $\left[\begin{array}{c}\frac{1}{\alpha^{3}} \\ \frac{1}{\alpha^{2}} \\ \frac{1}{\alpha} \\ 1\end{array}\right]$.

Similarly, for $\beta, \gamma$ and $\delta$, the corresponding eigenvectors are $\left[\begin{array}{c}\frac{1}{\beta^{3}} \\ \frac{1}{\beta^{2}}\end{array}\right],\left[\begin{array}{c}\frac{1}{\gamma^{3}} \\ \frac{1}{\gamma^{2}}\end{array}\right]$ and $\left[\begin{array}{l}\frac{1}{\delta^{3}} \\ \frac{1}{\delta^{2}} \\ \frac{1}{\delta} \\ 1\end{array}\right]$ respectively. Let $M=\left[\begin{array}{cccc}\left(\frac{1}{\alpha^{3}}\right) & \left(\frac{1}{\beta^{3}}\right) & \left(\frac{1}{\gamma^{3}}\right) & \left(\frac{1}{\delta^{3}}\right) \\ \left(\frac{1}{\alpha^{2}}\right) & \left(\frac{1}{\beta^{2}}\right) & \left(\frac{1}{\gamma^{2}}\right) & \left(\frac{1}{\delta^{2}}\right) \\ \left(\frac{1}{\alpha}\right) & \left(\frac{1}{\beta}\right) & \left(\frac{1}{\gamma}\right) & \left(\frac{1}{\delta}\right) \\ 1 & 1 & 1 & 1\end{array}\right]$ 
whose column vectors are the eigenvectors of $P$ corresponding to eigenvalues $\alpha, \beta, \gamma$ and $\delta$. Then by eigen decomposition of a matrix [3], we write

$$
P=M\left[\begin{array}{llll}
\alpha & 0 & 0 & 0 \\
0 & \beta & 0 & 0 \\
0 & 0 & \gamma & 0 \\
0 & 0 & 0 & \delta
\end{array}\right] M^{-1}
$$

This gives,

$$
P^{n}=M\left[\begin{array}{cccc}
\alpha^{n} & 0 & 0 & 0 \\
0 & \beta^{n} & 0 & 0 \\
0 & 0 & \gamma^{n} & 0 \\
0 & 0 & 0 & \delta^{n}
\end{array}\right] M^{-1} .
$$

By applying elementary row operations on $M$, we get
Binet - Type Formula For The Sequence of Tetranacci Numbers By Alternate Methods 


\section{Hathiwala, GS}

Shah, DV

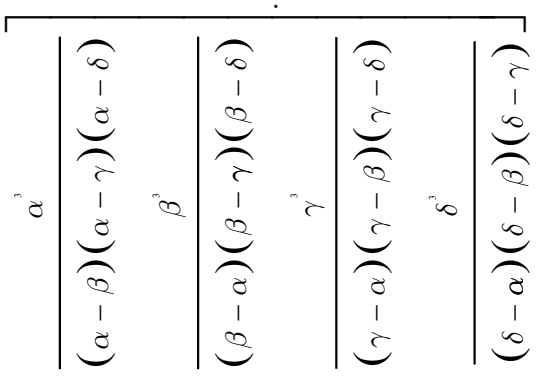

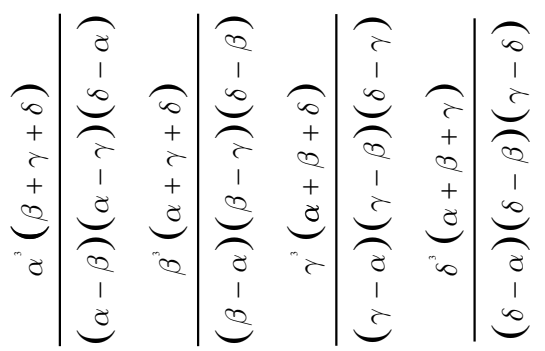
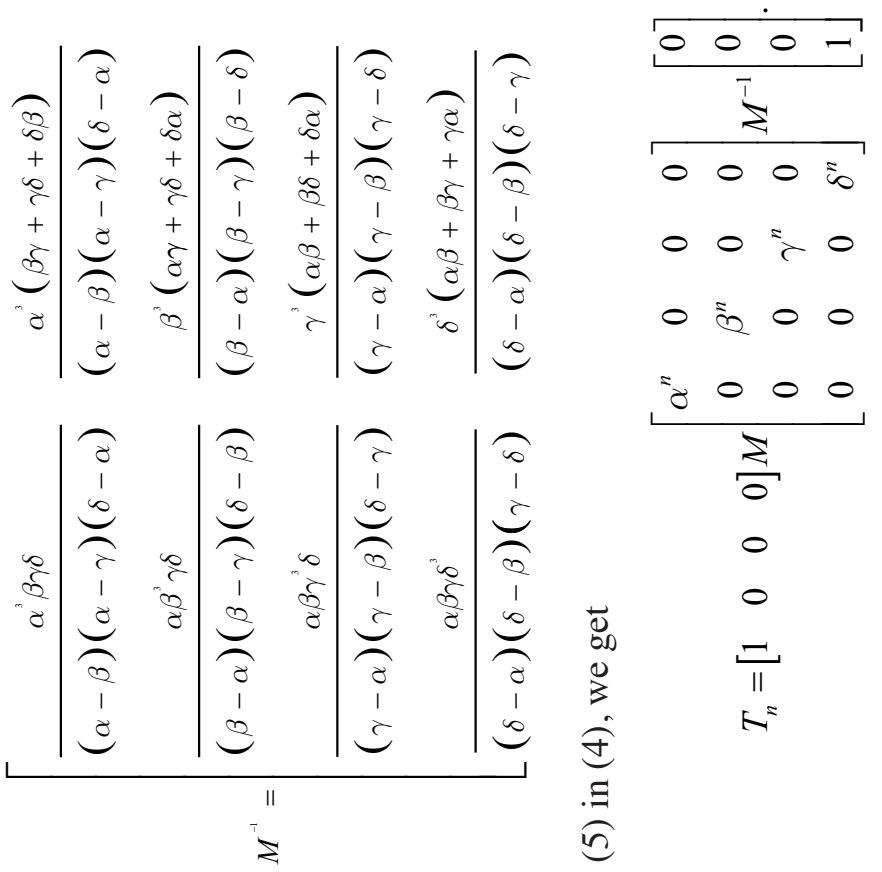


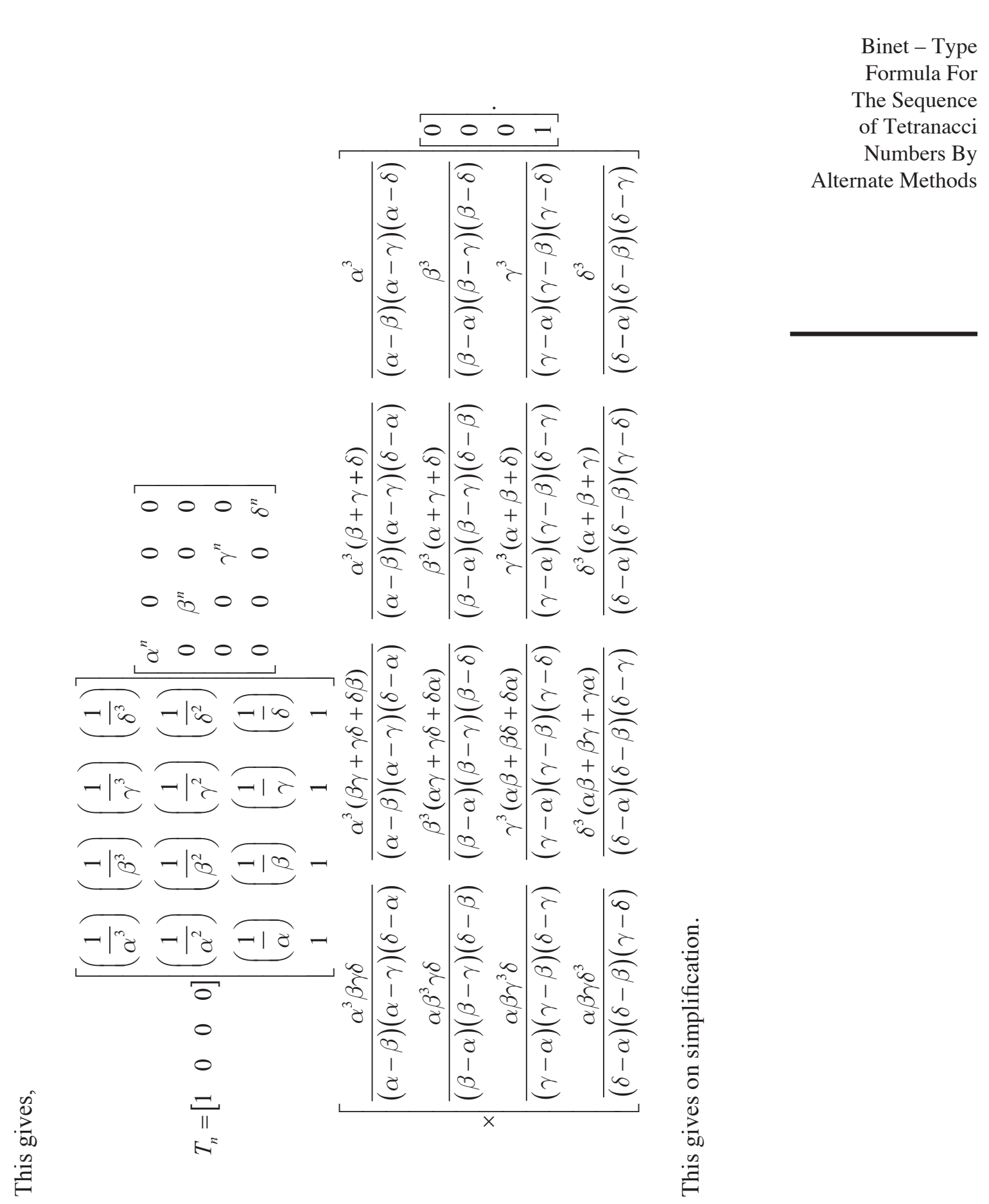


Hathiwala, GS Shah, DV

$$
\begin{aligned}
& T_{n}=\frac{\alpha^{n}}{(\alpha-\beta)(\alpha-\gamma)(\alpha-\delta)}+\frac{\beta^{n}}{(\beta-\alpha)(\beta-\gamma)(\beta-\delta)}+\frac{\gamma^{n}}{(\gamma-\alpha)(\gamma-\beta)(\gamma-\delta)} \\
& +\frac{\delta^{n}}{(\delta-\alpha)(\delta-\beta)(\delta-\gamma)}, \text { as required. }
\end{aligned}
$$

We next find the same result by the use of generating function for $T_{n}$.

$$
\text { Theorem 3.2: } \begin{aligned}
T_{n} & =\frac{\alpha^{n}}{(\alpha-\beta)(\alpha-\gamma)(\alpha-\delta)}+\frac{\beta^{n}}{(\beta-\alpha)(\beta-\gamma)(\beta-\delta)} ; \\
& +\frac{\gamma^{n}}{(\gamma-\alpha)(\gamma-\beta)(\gamma-\delta)}+\frac{\delta^{n}}{(\delta-\alpha)(\delta-\beta)(\delta-\gamma)}
\end{aligned}
$$

where $\alpha=1.97562, \beta=-0.774804, \gamma=-0.076379+0.8147 i$ and $\delta=\bar{\gamma}$.

Proof: By proposition 2.2, we have $t(x)=\frac{x^{3}}{1-x-x^{2}-x^{3}-x^{4}}$.

From theorem 3.1, we write

$$
\begin{gathered}
1-x-x^{2}-x^{3}-x^{4}=(1-\alpha x)(1-\beta x)(1-\gamma x)(1-\delta x) . \text { This gives } \\
t(x)=\frac{x^{3}}{(1-\alpha x)(1-\beta x)(1-\gamma x)(1-\delta x)} . \text { We now write } \\
\frac{x^{3}}{(1-\alpha x)(1-\beta x)(1-\gamma x)(1-\delta x)} \\
=\frac{A}{(1-\alpha x)}+\frac{B}{(1-\beta x)}+\frac{C}{(1-\gamma x)}+\frac{D}{(1-\delta x)} .
\end{gathered}
$$

Then $x^{3}=A(1-\beta x)(1-\gamma x)(1-\delta x)+B(1-\alpha x)(1-\gamma x)(1-\delta x)$

$$
+C(1-\alpha x)(1-\beta x)(1-\delta x)+D(1-\alpha x)(1-\beta x)(1-\gamma x) \text {. }
$$

If we consider $x=\frac{1}{\alpha}$, we get $\left(\frac{1}{\alpha}\right)^{3}=A\left(1-\frac{\beta}{\alpha}\right)\left(1-\frac{\gamma}{\alpha}\right)\left(1-\frac{\delta}{\alpha}\right)$. 
This gives $A=\frac{1}{(\alpha-\beta)(\alpha-\gamma)(\alpha-\delta)}$. Similarly, we get

$$
\begin{aligned}
& B=\frac{1}{(\beta-\alpha)(\beta-\gamma)(\beta-\delta)}, \\
& C=\frac{1}{(\gamma-\alpha)(\gamma-\beta)(\gamma-\delta)} \text { and } D=\frac{1}{(\delta-\alpha)(\delta-\beta)(\delta-\gamma)} .
\end{aligned}
$$

Thus (6) can be written as

$$
t(x)=A(1-\alpha x)^{-1}+B(1-\beta x)^{-1}+C(1-\gamma x)^{-1}+D(1-\delta x)^{-1}
$$

This gives $t(x)=A \sum_{i=0}^{\infty} \alpha^{i} x^{i}+B \sum_{i=o}^{\infty} \beta^{i} x^{i}+c \sum_{i=0}^{\infty} \gamma^{i} x^{i}+D \sum_{i=0}^{\infty} \delta^{i} x^{i}$.

Using the values $A, B, C$ and $D$, we get

$$
\begin{aligned}
t(x) & =\sum_{i=0}^{\infty}\left(\frac{\alpha^{i}}{(\alpha-\beta)(\alpha-\gamma)(\alpha-\delta)}+\frac{\beta^{i}}{(\beta-\alpha)(\beta-\gamma)(\beta-\delta)}\right) \\
& +\left(\frac{\gamma^{i}}{(\gamma-\alpha)(\gamma-\beta)(\gamma-\delta)}+\frac{\delta^{i}}{(\delta-\alpha)(\delta-\beta)(\delta-\gamma)}\right) x^{i} .
\end{aligned}
$$

But, from lemma 2.2, $t(x)=\sum_{n=0}^{\infty} T_{n} x^{n}$. Thus comparing coefficients on both sides, we get

$$
\begin{aligned}
T_{n} & =\frac{\alpha^{n}}{(\alpha-\beta)(\alpha-\gamma)(\alpha-\delta)}+\frac{\beta^{n}}{(\beta-\alpha)(\beta-\gamma)(\beta-\delta)} \\
& +\frac{\gamma^{n}}{(\gamma-\alpha)(\gamma-\beta)(\gamma-\delta)}+\frac{\delta^{n}}{(\delta-\alpha)(\delta-\beta)(\delta-\gamma)}
\end{aligned}
$$

\section{REFERENCES}

[1] Arfken, G.: Diagonalization of Matrices, Mathematical Methods for Physicists, 3rd ed. Orlando, FL: Academic Press, 1985, 217-229.

[2] Dresden G.P.B., Du Z.: A Simplified Binet Formula for Generalized Fibonacci Numbers, Journal of Integer Functions, Vol. 17, article 14.4.7, 2014.

[3] Eigen decomposition of a matrix:

http://mathworld.wolfram.com/EigenDecompositionTheorem.html 
Hathiwala, GS Shah, DV
[4] Hathiwala G. S., Shah D. V.: Golden proportions for the generalized Tetranacci numbers, International Research Journal of Mathematics, Enigneering and IT, Vol. 3, Issue 4, April 2016, 90-101.

[5] Hathiwala G. S., Shah D. V.: "Periodicity of Tetranacci Numbers Modulo", The Journal of the Indian Academy of Mathematics, Vol. 38, Issue 2, 2016, pp. 155-165, ISSN: $0970-5120$.

[6] Lee G. Y.,Lee G. S., Kim J.S., Shin H. K.: The Binet Formula and Representations of Generalized Fibonacci Numbers, Fibonacci Quarterly, Vol. 39, No. 2, May 2001, 158-164.

[7] Mehta D. A.: Ph.D. thesis entitled "Properties of the Sequences of Tribonacci Numbers and Generalized Cut - off Numbers", Veer Narmad South Gujarat University, Surat, India, Oct. 2009.

[8] Raab J.A.: A Generalization of the Connection between the Fibonacci Sequence and Pascal's Triangle, The Fibonacci Quarterly, Vol. 1, No. 3, Oct. 1963, 21-32.

[9] Singh B., Bhatnagar S., Sikhwal O.: Fibonacci - Like Sequence, International Journal of Advanced Mathematical Sciences, Vol. 1, No. 3, 2013, 145-151.

[10] Waddill M.E.: Some Properties of Tetranacci Numbers modulo, The Fibonacci Quarterly, Vol. 30, No. 3, Aug. 1992, 232-238.

[11] Waddill M.E.: The Tetranacci Sequence and Generalizations, The Fibonacci Quarterly, Vol. 30, No. 1, Feb. 1992, 9-20.

[12] Wall D. D.: Fibonacci Series Modulo , American Math. Monthly, Vol. 67, 1960, 525-532. 